\title{
The landscape configuration of zoonotic transmission of Ebola virus disease in West and Central Africa: Interaction between population density and vegetation cover
}

Michael Walsh, M.A. Haseeb

Ebola virus disease (EVD) is an emerging infectious disease of zoonotic origin that has been responsible for high mortality and significant social disruption in West and Central Africa. Zoonotic transmission of EVD requires contact between susceptible human hosts and the reservoir species for Ebolaviruses, which are believed to be fruit bats.

Nevertheless, features of the landscape that may facilitate such points of contact have not yet been adequately identified. Nor have spatial dependencies between zoonotic EVD transmission and landscape structures been delineated. This investigation sought to describe the spatial relationship between zoonotic EVD transmission events, or spillovers, and population density and vegetation cover. An inhomogeneous Poisson process model was fitted to all precisely geolocated zoonotic transmissions of EVD in West and Central Africa. Population density was strongly associated with spillover, however there was significant interaction between population density and green vegetation cover. In areas of very low population density, increasing vegetation cover was associated with a decrease in risk of zoonotic transmission, but as population density increased in a given area, increasing vegetation cover was associated with increased risk of zoonotic transmission. This study showed that the spatial dependencies of Ebolavirus spillover were associated with the distribution of population density and vegetation cover in the landscape, even after controlling for climate and altitude. While this is an observational study, and thus precludes direct causal inference, the findings do highlight areas that may be at risk for zoonotic EVD transmission based on the spatial configuration of important features of the landscape. 
1 The landscape configuration of zoonotic transmission of Ebola virus disease in West

2 and Central Africa: Interaction between population density and vegetation cover.

3 Michael G. Walsh ${ }^{1}$ and M.A. Haseeb ${ }^{1,2}$

$4{ }^{1}$ Department of Epidemiology and Biostatistics, School of Public Health,

$5 \quad 1^{2}$ Departments of Cell Biology, Pathology and Medicine, College of Medicine, State

6 University of New York, Downstate Medical Center, Brooklyn, New York, U.S.A.

7 Address correspondence to:

8 Michael Walsh, PhD, MPH

9 Assistant Professor, Epidemiology and Biostatistics

10 School of Public Health

11 State University of New York, Downstate

12450 Clarkson Avenue, Box 43

13 Brooklyn, NY 11203

14 thegowda@gmail.com, michael.walsh@downstate.edu

15 Phone: (347) 557-1108

16 Introduction 
17 Ebola virus disease (EVD) first emerged in 1976 in what are today the Democratic

18 Republic of the Congo and South Sudan. Over the course of the last 4 decades there

19 have been a total of 24 outbreaks in 10 countries in Central and West Africa. The 20 case fatality associated with EVD outbreaks is typically high ( mean $=65 \%$; range $=$

$2132 \%-90 \%$ ) causing fear and social disruption when they occur. Never has this been

22 more apparent than during the 2014 EVD outbreak in West Africa, which is currently

23 underway. This outbreak is the largest documented EVD event in history and has

24 affected the populations of Guinea, Sierra Leone, Liberia, and Nigeria with 10141

25 confirmed, probable, and suspected cases and 4922 deaths as of 24 October, 2014

26 according to the Centers for Disease Control and Prevention case counts (CDC,

27 2014). Because of the increasing magnitude of the burden of EVD, locating sources

28 of human outbreaks is an important public health priority. Ebolaviruses require two

29 transmission processes for outbreaks to emerge. First, there must be a spillover

30 event, which is defined as a zoonotic transmission from either the primary sylvan

31 reservoir host (e.g. fruit bats) or from a secondary sylvan host for whom the virus is

32 also pathogenic (e.g. non-human primates) (Allaranga et al., 2010). Second, once

33 the spillover generates the index case(s), subsequent person-to-person spread

34 follows the care of the affected individuals via contact with contaminated body fluids

35 (Allaranga et al., 2010). As the upstream source of all subsequent human-to-human

36 transmission, spillover is thus an important antecedent to widespread human

37 outbreaks. Nevertheless, despite four decades of EVD outbreak management, as

38 well as research into the epidemiology and infection ecology of Ebolaviruses, we still

39 have yet to clearly delineate the biologic, physical, and social features of the

40 landscape that defines zoonotic infection risk. More specifically, the collective

41 experience of these twenty-four outbreaks have not yet fully revealed the landscape

42 epidemiology of spillover events. Locating potential points of spillover by

43 recognizable features in the landscape may prove useful in blocking zoonotic 
44 transmission to humans and thus preventing human outbreaks, which are

45 notoriously difficult to control in the ensuing social disruption, before they happen.

46 For example, ecologic, economic, and social disruption, which may lead to displaced

47 human and animal populations with emerging pressures of deforestation and

48 encroachment, have often been posited as determinants of EVD outbreaks (Bausch

49 \& Schwarz, 2014; Pourrut et al., 2005). Poverty is also an important driver of human-

50 animal interaction (Brashares, Golden, Weinbaum, Barrett, \& Okello, 2011).

51 However, while certainly ecologically plausible and supported by a few small local

52 field investigations (Daszak, Cunningham, \& Hyatt, 2000; Georges et al., 1999; Leroy

53 et al., 2004; Wolfe, 2005), this remains theoretical across much of the region where

54 these outbreaks occur (Daszak et al., 2000; McCallum \& Dobson, 1995). Moreover,

55 while one recent investigation has provided valuable insight into the ecologic niche

56 of EVD (Pigott et al., 2014), no studies to date have considered the collective

57 experience of EVD as an explicitly spatial process. The current investigation sought

58 to explore the role of human population density and vegetation cover on the spatial

59 distribution and risk of zoonotic transmission of EVD. The historical and current

60 experience of these EVD outbreaks from 1976 to 2014 was used to test the

61 hypothesis that increasing human population density in forested landscapes would

62 be associated with increased zoonotic transmission. This investigation does not

63 assess human to human transmission dynamics and subsequent epidemics, but

64 rather focuses specifically on isolated spillover events.

65 Methods

66 The PubMed database maintained by the National Library of Medicine was queried

67 for all primary reports of outbreaks of Ebola virus disease (EVD) beginning with the

68 initial outbreaks in the Democratic Republic of the Congo (previously Zaire) and

69 South Sudan (previously Sudan) in 1976 and ending with the 2014 West Africa 
70 outbreak, which began in Guinea in 2013, and the 2014 outbreak in the Democratic

71 Republic of the Congo. In addition, all archives of the World Health Organization's

72 (WHO) Weekly Epidemiological Record and other WHO reports were queried to

73 identify any additional EVD outbreaks or individual cases that were not represented

74 in the peer-reviewed scientific literature. A total of 36 reports were obtained (peer-

75 reviewed manuscripts $=27$; WHO reports $=9$ ). From these reports the index case(s)

76 was identified from each outbreak and the geographic location recorded at the

77 village level. Each of the 24 outbreaks, with their 32 distinct spillover events and

78 locations, and all references are listed in Supplementary Table 1. The locations of

79 zoonotic transmission events were assigned geographic coordinates using longitude

80 and latitude as the coordinate reference system and obtained in Google Maps and

81 cross referenced with Open Street Map [openstreetmap.org]. Only 21 of the 24

82 zoonotic EVD outbreaks (or 29 of 32 unique spillover events) were included in this

83 analysis. Three spillover events were not considered in this analysis because either

84 the precise location was not identified in the outbreak reports (Luwero District,

85 Uganda, 2012-2013 and Isiro, DRC, 2012), or could not be geolocated (Nakisamata,

86 Uganda, 2011). Given that this investigation is attempting to understand the

87 occurrence of EVD spillover as a point process, it was deemed inappropriate to treat

88 these three events as polygons in the analysis.

89 Climate data were obtained from the bioclimatic data maintained by the WorldClim

90 Global Climate database (Data, n.d.). Specifically, the annual mean temperature and

91 annual mean precipitation from 1950 to 2000 were each extracted as 30 arc second

92 resolution rasters (Hijmans, Cameron, Parra, Jones, \& Jarvis, 2005). A raster for

93 altitude at the same resolution was also obtained. Each pixel in these rasters

94 represents the annual mean temperature, annual mean precipitation, and altitude

95 for that approximately 1 square kilometer point on the Earth's surface. 
96 Similarly, we acquired a raster for the MODIS-based Maximum Green Vegetation

97 Fraction (MGVF) from the United States Geologic Survey's Land Cover Institute

98 (Institute, n.d.). This raster describes the percentage of green vegetation cover per

99 pixel and is a function of the normalized difference vegetation index (Broxton, Zeng,

100 Scheftic, \& Troch, 2014). The MGVF raster resolution is, again, approximately 1

101 kilometer squared.

102 Finally, human population density was obtained from the Socioeconomic Data and

103 Applications Center (SEDAC), which is part of the National Aeronautics and Space

104 Agency's Earth Observing System Data and Information System (Center for

105 International Earth Science Information Network - CIESIN - Columbia University,

106 International Food Policy Research Institute - IFPRI, The World Bank, n.d.). Population

107 density is represented by a 30 arc-second raster derived from the Global Rural-

108 Urban Mapping Project estimates for the 2000 population, where each pixel

109 represents the population density for that approximately 1 square kilometer point on

110 the Earth's surface (Balk et al., 2006).

111 Statistical Methods

112 The 29 geolocated zoonotic EVD spillover events were modeled as a point process

113 (Baddeley \& Turner, 2000) across the tropical belt of narrow latitude and wide

114 longitude in West and Central Africa. The specific geographic extent of the

115 investigation was latitude $40^{\circ} \mathrm{S}, 35^{\circ} \mathrm{N}$, and longitude $20^{\circ} \mathrm{W}, 60^{\circ} \mathrm{E}$.

116 First, as a null model indicating complete spatial randomness, the outbreaks were

117 considered as a homogeneous Poisson process with conditional intensity,

$$
\lambda(u, X)=\beta
$$

119 where $u$ represents the set of locations corresponding to the pattern of points, X, 120 and $\beta$ is the intensity parameter. With this formulation, the expected number of 
121 points (i.e. intensity) in any subregion of the larger geographic extent is simply

122 proportional to the area of that subregion (Baddeley \& Turner, 2000).

123 Subsequently, the model representing a homogeneous Poisson process was

124 compared to a model representing an inhomogeneous Poisson process, which

125 indicates a spatial dependency in the pattern of zoonotic transmisison, and has

126 conditional intensity,

$$
\lambda(u, X)=\beta(u)
$$

128 and now shows the intensity as a function of the location, $u$, of the points. Given the 129 better model fit of the inhomogeneous Poisson process (see Results), the

130 investigation proceeded under the assumption that the intensity was spatially

131 dependent and, thus, attempted to identify those landscape features that were

132 associated with the spatial distribution of zoonotic transmission events in West and

133 Central Africa. These features were included in the inhomogeneous point process

134 model as spatially explicit covariates with conditional intensity,

$$
\lambda(u, X)=\rho(Z(u))
$$

136 where $\rho$ is the function describing the association between the point intensity and

137 the set of covariates $\mathbf{Z}$ at location $u$.

138 Six covariates corresponding to the landscape features of interest were considered

139 in this analysis: population density, MGVF, mean temperature, mean precipitation,

140 altitude, and an interaction covariate between population density and MGVF.

141 Population density is presented in the results in increments of 100 persons $/ \mathrm{km}^{2}$, and

142 altitude is presented in 10 meter increments. The association between the intensity

143 of zoonotic EVD transmission events and each covariate was assessed via the 
144 relative risks derived from the regression coefficients of the inhomogeneous Poisson

145 model. First, a homogeneous Poisson "null" model was fit and compared to an

146 inhomogeneous model, which specified only spatial trend. Subsequently, an

147 inhomogeneous model with the covariates described above was fitted to the EVD

148 spillover events to identify possible sources of spatial dependency. The covariates in

149 the full model assess the independent association between intensity and each

150 landscape factor adjusted for all other factors, while simultaneously assessing the

151 interaction between population density and MGVF. We did also consider both

152 temperature range and isothermality in our model. However, these contributed to

153 multicollinearity with mean temperature leading to model instability and so, as

154 these were not significantly associated with EVD spillover events (RR $=1.0,95 \%$ C.I.

$1550.99-1.00$ for both together and independently), these were not included in the

156 final analysis. The R language was used for all analyses (http://www.r-project.org/).

157 The ppm function in the spatstat package was used for the point process model, and

158 the valid.ppm function was used to verify that the fitted models specified well-

159 defined point processes (Baddeley \& Turner, 2005). The R code for these models is 160 presented in Supplementary File 1.

161 Results

162 The geographic distribution of the 29 precisely geolocated zoonotic transmission

163 events that occurred in West and Central Africa between 1976 and 2014 are

164 depicted in the map in Figure 1. Comparison of the homogeneous and

165 inhomogeneous Poisson process models based on the likelihood ratio test $(p<0.05)$

166 suggests that zoonotic EVD transmission is spatially dependent.

167 Table 1 presents the regression coefficients from the inhomogeneous Poisson

168 process model, which represents the adjusted measures of association between

169 zoonotic EVD and each covariate. That is, the measure of association for each 
170 landscape factor is adjusted for all others in the model. Both population density (RR

$171=0.98,95 \%$ C.I. $0.97-0.99)$ and MGVF (RR $=0.99,95 \%$ C.I $0.94-1.05)$ were

172 inversely associated with the spatial distribution of zoonotic transmission events,

173 wherein increasing population density or vegetation cover, respectively,

174 corresponded to decreasing spillover risk. However, given that this model assessed

175 the association between zoonotic EVD and the landscape factors population density

176 and MGVF, each as modifying the other, we must also consider the significant

177 interaction between them $(R R=1.0002,95 \%$ C.I. $1.0001-1.0003)$ to arrive at the

178 correct interpretation. The interaction term describes how the relationship between

179 zoonotic EVD and each of the two landscape factors changes at different levels of

180 the other. For example, at a population density of 0 persons $/ \mathrm{km}^{2}$ the association

181 between vegetation cover is simply the RR for MGVF, where each percentage

182 increase in cover corresponded to a $2 \%$ decrease in spillover risk. However, each

183100 persons $/ \mathrm{km}^{2}$ increase in population density alters the association between MGVF

184 and zoonotic EVD by a factor of $2 \%(R R=1.02 \times 0.99=1.0098)$, which corresponds

185 to a diminishing protective effect of vegetation cover as the population density for

186 that area increases. Indeed, vegetation cover is no longer protective at a population

187 density of just 100 persons $/ \mathrm{km}^{2}$. A threshold of population density is reached at 200

188 persons $/ \mathrm{km}^{2}$ after which zoonotic transmission risk is greater than $1 \%$ with each $1 \%$

189 increase in vegetation cover $\left(R R=1.02^{2} \times 0.99=1.03\right)$. Similar effect modification

190 of population density by MGVF is implied. In addition, temperature and altitude were

191 both associated with zoonotic EVD with each $1^{\circ}$ increase in temperature and 10

192 meter increase in altitude corresponding to a $7 \%$ and $4 \%$ decrease in risk,

193 respectively. The point process was verified as being well-defined for the fitted

194 model, and the likelihood ratio test comparing the interaction model to a no

195 interaction model suggested that the former was the better fit $(p<0.01)$ 
196 Figure 2 displays maps of the distribution of temperature, altitude, vegetation cover,

197 and human population density across the African continent with the zoonotic

198 transmission event points superimposed. All landscape factors show some level of

199 heterogeneity across much of the range of zoonotic EVD transmission, but

200 population density appears much more heterogeneous in space. Figure 3 maps the

201 predicted intensity of spillover as a spatially dependent function of the landscape

202 features in the inhomogeneous Poisson model. Red areas in the map represent areas

203 where spillover events are predicted by the model. The map predicts spillover in

204 areas of Gabon, the Republic of Congo, Uganda, and some smaller regions of the

205 DRC, all countries which have seen significant outbreaks in the past. However, there

206 are also some areas with predicted intensity in countries without previous spillover

207 events such as Rwanda, Burundi, Cameroon, the Central African Republic, Nigeria,

208 Ghana, and Cote d'Ivoire. It is important to note that the predicted spillover in all of

209 these countries is highly area specific, with risk being determined by the spatial

210 configuration of population density, vegetation cover, temperature, and altitude.

\section{Discussion}

212 This study explored the spatial dependence of 29 known EVD spillover events on

213 specific landscape features within the geographic range where these events have

214 occurred on the African continent. The interaction between human population

215 density and vegetation cover was the single most important feature associated with

216 the occurrence of zoonotic transmission. In the absence of population pressure,

217 increasing vegetation cover was associated with decreased risk of zoonotic

218 transmission. However, as population density increased across geographic space in

219 the presence of higher vegetation coverage, the protective effect of MGVF was

220 reversed and ultimately a threshold is reached at 200 persons $/ \mathrm{km}^{2}$ after which

221 zoonotic transmission risk is greater than 1\% with each $1 \%$ increase in vegetation 


\section{PeerJ Reviewing Manuscript}

222 cover. These results suggest that the interaction between human population density

223 and sylvan habitat may create pressure in the landscape that open conduits to EVD

224 spillover into human communities.

225 It has been postulated that human encroachment on sylvan habitats is an important

226 mediating factor in the emergence of novel infectious diseases in humans (Daszak

227 et al., 2000; Estrada-Peña, Ostfeld, Peterson, Poulin, \& de la Fuente, 2014; Wolfe,

228 Dunavan, \& Diamond, 2007; Woolhouse \& Gowtage-sequeria, 2005), and in the

229 emergence of EVD in particular (Bausch \& Schwarz, 2014; Wolfe, 2005). More

230 specifically, the repurposing of sylvan landscapes for agricultural, industrial, and

231 residential land use frequently coincides with altered population densities that result

232 from concentrated abrupt events such as conflict, or from the more diffuse and

233 insidious processes of inequitable expansion of global and local economies (Bausch

234 \& Schwarz, 2014; Wolfe et al., 2007). Typically, varying levels of forest

235 fragmentation then accrue. This fragmentation often presents anthropogenic

236 ecotones that have the potential to act as conduits for human-animal interaction,

237 where, previously, such contact was blocked by intact sylvan ecosystems (Smith \&

238 Smith, 2001). Moreover, shifting forms of land use among isolated populations, such

239 as the location and extent of subsistence hunting, are often driven by poverty and

240 inequitable economic development (Brashares et al., 2011). As such, where nascent

241 human-animal interactions facilitate the transmission of novel pathogens from

242 sylvan reservoirs to incidental human hosts, outbreaks of emerging infections may

243 follow (Georges et al., 1999; Leroy et al., 2004; Wolfe, 2005). Nevertheless, very few

244 investigations have attempted to measure the specific association between human

245 influence in the landscape and the spillover of Ebolaviruses from animal to human

246 populations. The current investigation was the first to attempt to quantify zoonotic

247 transmission risk as spatially dependent on human-forest interaction across the 
248 complete zoonotic EVD experience in West and Central Africa. In one excellent

249 recent study, investigators used an ecologic niche approach to identify the

250 geographic range of Ebolavirus presence, and thus recognize areas of potential

251 spillover to humans (Pigott et al., 2014). Their species distribution models used

252 climate data, land cover, and the observed presence of presumed Ebolavirus

253 reservoir bat species to model the presence or absence of Ebolaviruses based on all

254 documented human and animal EVD outbreaks. The result is a nice delineation of

255 the ecologic niche of Ebolaviruses across sub-Saharan Africa, the extent of which

256 largely coincides with the predicted risk in the current study. However, the ecologic

257 niche of the former study did not directly describe the relationship between human

258 pressure in specific landscapes and zoonotic transmission, nor did it model the

259 zoonotic transmission events explicitly as a point process with spatially dependent

260 intensity. As such the current study adds to the previous work by highlighting areas

261 of particular zoonotic transmission risk due to the spatial configuration of the

262 interaction between human population density and vegetation cover in the

263 landscape. It is also worth noting that, by itself, vegetation cover was not strongly

264 associated with EVD spillover while population density was. Nevertheless, the

265 relevance of vegetation cover is more apparent in its interaction with population

266 density. We feel this also makes sense intuitively since, by itself, an area of intact

267 forest with dense vegetation cover could conceivably either elevate or decrease risk.

268 Presumably, one would be more likely to encounter the natural reservoir for an

269 Ebolavirus while in a pristine sylvan habitat and, as such, it may appear that dense

270 vegetation cover confers risk. However, such a habitat may also be less likely to

271 have people moving through it, which may give the appearance of diminishing risk.

272 Conversely an urban community with much less vegetation cover would presumably

273 be less likely to harbor the reservoir species and more likely to suppress human-

274 animal encounters, and thus higher population density may appear to absorb risk. 


\section{PeerJ Reviewing Manuscript}

275 However, the actual risk of spillover ultimately depends on the potential for those

276 human-animal encounters, which would require a minimum presence of sylvan

277 space and human presence in close proximity to that space. The results of the

278 current study support such a relationship.

279 This study also identified an inverse relationship between zoonotic EVD transmission

280 and both temperature and altitude. Increases in both temperature and altitude

281 corresponded to lower spillover risk, which conforms to the previously mentioned

282 study by Pigott et al. for altitude but not temperature (Pigott et al., 2014). Increasing

283 altitude may reduce the necessary human-animal encounters because of climatic

284 changes (i.e. reduced temperature) or plant and animal species distribution changes

285 that attend changes in elevation, or because of lower population density at altitude.

286 It is unclear why increasing temperatures were associated with lower spillover

287 intensity in this study. It may be that areas of higher mean temperature were also

288 areas of lower minimum temperatures, as is the case in more northern latitudes

289 entering into the African Sahel, which, to date, has also demarcated the northern

290 boundary of Ebolavirus transmission. Moreover, such a boundary may in fact be due

291 to differences in the temperature range, or may simply be due to a lack of the

292 reservoir species range extending that far north (Pigott et al., 2014).

293 There are some important limitations inherent in this study. First, the rasters for

294 temperature and precipitation consisted of single composite measures over the 295 period 1950 to 2000 . In addition, the earliest available raster for MGVF was from

296 2001. As such, while these three rasters exhibited high spatial resolution $(\sim 1$

297 square kilometer), the temporal resolution was coarse, given that the former two 298 were averaged over a 50 year time span and the latter was a single measurement.

299 Nevertheless, we feel that the measures of temperature and precipitation in this 300 study are more indicative of the general climate of the regions represented, and 


\section{PeerJ Reviewing Manuscript}

301 thus provide a more robust approach to controlling for background climate while

302 assessing the impact of human influence. These climate measures would be

303 expected to be less accurate for assessing more nuanced patterns in weather on the

304 occurrence of zoonotic EVD transmission. In addition, the measure of MGVF, which

305 was captured in this raster in 2001, was taken roughly at the median year (2002) of

306 all EVD outbreaks and, as such, can be considered an approximation of green

307 vegetation cover at the temporal midpoint of the zoonotic transmission point

308 process. Again, while such a measure may not be sufficient for assessing the effect

309 of complex change in vegetation cover over time, we feel that, as a measure of the

310 central tendency of MGVF, it is an appropriate representation of the background

311 vegetation cover while assessing human population density at the same time

312 period. Second, the number of occurrences comprising the full history of

313 documented EVD outbreaks in Africa is relatively small. As such, the sample size for

314 this investigation is also small, with only 29 precisely geolocated spillover events

315 available. Therefore, this collection of zoonotic events may not be representative of

316 the total potential events in the region, and the model may overestimate risk for

317 landscape features and geographic areas with a high relative occurrence and

318 underestimate risk for landscape features and geographic areas with a low relative

319 occurrence. Third, as described above in the Statistical Methods section,

320 temperature range and isothermality were excluded from the model due to

321 multicollinearity. As such, these are two climatic factors that were not included in

322 the inhomogeneous Poisson model and, thus, predictions of spillover risk are less

323 reflective of climate nuance than they would be if these two factors were included.

324 Nevertheless, as mentioned above, neither temperature range nor isothermality

325 were significantly associated with EVD spillover events (RR $=1.0,95 \%$ C.I. 0.99 -

3261.00 for both) so we expect that their influence on predicted spillover risk is not

327 dramatic. Finally, this is fundamentally an observational study, which precludes any 
328 direct causal interpretation of the findings. The associations described are just that,

329 observational associations. They may suggest relationships between features of the

330 landscape and zoonotic EVD risk, but they do not definitively identify what could be

331 called true causal relationships. The latter can only be discerned by direct

332 measurement of animals and humans in the specific locations where zoonotic

333 transmission events are taking place. This will require extensive field investigation

334 incorporating human and animal serology, habitat description at a much finer

335 resolution and richer detail (i.e. describing not simply vegetation cover, but

336 providing more precise descriptions of habitat parcels in terms of biologic and

337 physical properties), and specific cultural and economic practices that bring humans

338 into close proximity to reservoir species. Moreover, identification of external sources

339 of local population movement and displacement may be particularly useful in

340 understanding the ways in which population density interacts with sylvan

341 landscapes to confer risk(Bausch \& Schwarz, 2014). Such research is an expensive

342 prospect indeed, but it is necessary if we are to generate sufficiently rich data to

343 inform actionable prevention structures that have real potential to limit dangerous

344 human-animal encounters and block zoonotic EVD transmission.

345 In conclusion, the current investigation identified a strong association between EVD

346 spillover and population density and effect modification with vegetation cover. The

347 results also suggest relationships with temperature and altitude, though these may

348 be incidental to the as yet unknown range of the reservoir species. While these

349 findings cannot be interpreted as causal due to the observational nature of the data,

350 they do suggest that the specific landscape configuration of interaction between

351 human populations and forested land may facilitate zoonotic EVD transmission. 
353

354

355

356

357

358

359

360

361

362

363

364

365

366

367

368

369

370

371

372

373

374

375

376

377

378

379

380

Allaranga, Y., Kone, M. L., Formenty, P., Libama, F., Boumandouki, P., Woodfill, C. J. I., ... Yada, A. (2010). Lessons learned during active epidemiological surveillance of Ebola and Marburg viral hemorrhagic fever epidemics in Africa. East African journal of public health, 7(1), 30-6. Retrieved from http://www.ncbi.nlm.nih.gov/pubmed/21413569

Baddeley, A., \& Turner, R. (2000). Practical Maximum Pseudolikelihood for Spatial Point Patterns (with Discussion). Australian <html_ent glyph="@amp;" ascii=" "\&"/> New Zealand Journal of Statistics, 42(3), 283-322. doi:10.1111/1467-842X.00128

Baddeley, A., \& Turner, R. (2005). spatstat: An R Package for Analyzing Spatial Point Patterns. Journal of Statistical Software 12(6). Retrieved from http://www.jstatsoft.org/v12/i06/

Balk, D. L., Deichmann, U., Yetman, G., Pozzi, F., Hay, S. I., \& Nelson, A. (2006). Determining global population distribution: methods, applications and data. Advances in parasitology, 62, 119-56. doi:10.1016/S0065-308X(05)62004-0

Bausch, D. G., \& Schwarz, L. (2014). Outbreak of Ebola Virus Disease in Guinea: Where Ecology Meets Economy. PLoS Neglected Tropical Diseases, 8(7), e3056. doi:10.1371/journal.pntd.0003056

Brashares, J. S., Golden, C. D., Weinbaum, K. Z., Barrett, C. B., \& Okello, G. V. (2011). Economic and geographic drivers of wildlife consumption in rural Africa. Proceedings of the National Academy of Sciences of the United States of America, 108(34), 13931-6. doi:10.1073/pnas.1011526108

Broxton, P. D., Zeng, X., Scheftic, W., \& Troch, P. A. (2014). A MODIS-Based Global 1$\mathrm{km}$ Maximum Green Vegetation Fraction Dataset. Retrieved from http://journals.ametsoc.org/doi/abs/10.1175/JAMC-D-13-0356.1

CDC. (2014). 2014 Ebola Outbreak in West Africa | Ebola Hemorrhagic Fever | CDC. Retrieved October 28, 2014, from http://www.cdc.gov/vhf/ebola/outbreaks/2014west-africa/index.html 
381

382

383

384

385

386

387

388

389

390

391

392

393

394

395

396

397

398

399

400

401

402

403

404

405

406

407

408

409

410

411

412

413

414

415

416

417

Center for International Earth Science Information Network - CIESIN - Columbia University, International Food Policy Research Institute - IFPRI, The World Bank, and C. I. de A. T.-C. (n.d.). Population Density Grid, v1: Global Rural-Urban Mapping Project (GRUMP), v1 | SEDAC. Palisades, NY: NASA Socioeconomic Data and Applications Center (SEDAC). Retrieved October 23, 2014, from http://sedac.ciesin.columbia.edu/data/set/grump-v1-population-density

Daszak, P., Cunningham, A. A., \& Hyatt, A. D. (2000). Emerging infectious diseases of wildlife--threats to biodiversity and human health. Science (New York, N.Y.), 287(5452), 443-9. Retrieved from

http://www.ncbi.nlm.nih.gov/pubmed/10642539

Data, W.-G. C. (n.d.). Data for current conditions ( 1950-2000)| WorldClim - Global Climate Data. WorldClim - Global Climate Data. Retrieved October 23, 2014, from http://www.worldclim.org/current

Estrada-Peña, A., Ostfeld, R. S., Peterson, a T., Poulin, R., \& de la Fuente, J. (2014). Effects of environmental change on zoonotic disease risk: an ecological primer. Trends in parasitology, 30(4), 205-14. doi:10.1016/j.pt.2014.02.003

Georges, A. J., Leroy, E. M., Renaut, A. A., Benissan, C. T., Nabias, R. J., Ngoc, M. T., ... Georges-Courbot, M. C. (1999). Ebola hemorrhagic fever outbreaks in Gabon, 1994-1997: epidemiologic and health control issues. The Journal of infectious diseases, 179 Suppl , S65-75. doi:10.1086/514290

Hijmans, R. J., Cameron, S. E., Parra, J. L., Jones, P. G., \& Jarvis, A. (2005). Very high resolution interpolated climate surfaces for global land areas. International Journal of Climatology, 25(15), 1965-1978. doi:10.1002/joc.1276

House, T. (2014). Epidemiological Dynamics of Ebola Outbreaks. eLife, 3, e03908. doi:10.7554/eLife.03908

Institute, T. U. L. C. (n.d.). MODIS-based Maximum Green Vegetation Fraction. Retrieved October 23, 2014, from http://landcover.usgs.gov/green_veg.php

Leroy, E. M., Rouquet, P., Formenty, P., Souquière, S., Kilbourne, A., Froment, J.-M., ... Rollin, P. E. (2004). Multiple Ebola virus transmission events and rapid decline of central African wildlife. Science (New York, N.Y.), 303(5656), 387-90. doi:10.1126/science.1092528

McCallum, H., \& Dobson, A. (1995). Detecting disease and parasite threats to endangered species and ecosystems. Trends in ecology \& evolution, 10(5), 1904. Retrieved from http://www.ncbi.nlm.nih.gov/pubmed/21237000

Pigott, D. M., Golding, N., Mylne, A., Huang, Z., Henry, A. J., Weiss, D. J., ... Hay, S. I. (2014). Mapping the zoonotic niche of Ebola virus disease in Africa. eLife, 3(December 2013), 1-29. doi:10.7554/eLife.04395 
418 Pourrut, X., Kumulungui, B., Wittmann, T., Moussavou, G., Délicat, A., Yaba, P., ... Leroy, E. M. (2005). The natural history of Ebola virus in Africa. Microbes and

420 infection / Institut Pasteur, 7(7-8), 1005-14. doi:10.1016/j.micinf.2005.04.006

421 Smith, R. L., \& Smith, T. M. (2001). Ecology and Field Biology: Hands-on Field

422 Package (Vol. 2001, p. 720). Pearson College Division. Retrieved from

423 http://books.google.com/books?id=E9MEAAAACAAJ\&pgis=1

424 Wolfe, N. D. (2005). Bushmeat Hunting, Deforestation, and Prediction of Zoonotic 425 Disease Emergence. Emerging infectious diseases, 11(12), 1822-1827.

426 doi:10.3201/eid1112.040789

427 Wolfe, N. D., Dunavan, C. P., \& Diamond, J. (2007). Origins of major human infectious 428 diseases. Nature, 447(7142), 279-83. doi:10.1038/nature05775

429 Woolhouse, M. E. J., \& Gowtage-sequeria, S. (2005). Host Range and Emerging and $430 \quad$ Reemerging Pathogens, 11(12). 
Table $\mathbf{1}_{\text {(on next page) }}$

Adjusted relative risks and $95 \%$ confidence intervals and $p$-values for the associations between zoonotic transmission of Ebola virus disease and features of the landscape.

These measures of association are derived from an inhomogeneous Poisson model of the point process of zoonotic transmission events with interaction between population density and maximum green vegetation fraction (MGVF). 


\begin{tabular}{|l|c|c|c|}
\hline Landscape Factor & $\begin{array}{c}\text { Relative } \\
\text { Risk* }\end{array}$ & $\begin{array}{c}95 \% \text { Confidence } \\
\text { Interval }\end{array}$ & P-value \\
\hline $\begin{array}{l}\text { Population density (100 } \\
\text { persons/km²) }\end{array}$ & 0.98 & $0.97-0.99$ & $<0.0001$ \\
\hline $\begin{array}{l}\text { Mean Green Vegetation Fraction } \\
\text { (MGVF) (\%) }\end{array}$ & 0.99 & $0.94-1.05$ & $>0.1$ \\
\hline Mean Temperature (C $\left.{ }^{\circ}\right)$ & 0.93 & $0.88-0.98$ & $<0.001$ \\
\hline Mean Precipitation (cm) & 1.00 & $0.99-1.001$ & $>0.1$ \\
\hline Altitude (10 meters) & 0.96 & $0.94-0.99$ & $<0.01$ \\
\hline $\begin{array}{l}\text { Population density: MGVF } \\
\text { interaction }\end{array}$ & 1.0002 & $1.0001-1.0003$ & $<0.0001$ \\
\hline
\end{tabular}

*The relative risks reflect the percent change in zoonotic transmission risk associated with each unit change (as listed in parentheses) in the corresponding landscape factor. 
The distribution of zoonotic Ebola virus disease transmission events (red dots) across West and Central Africa.

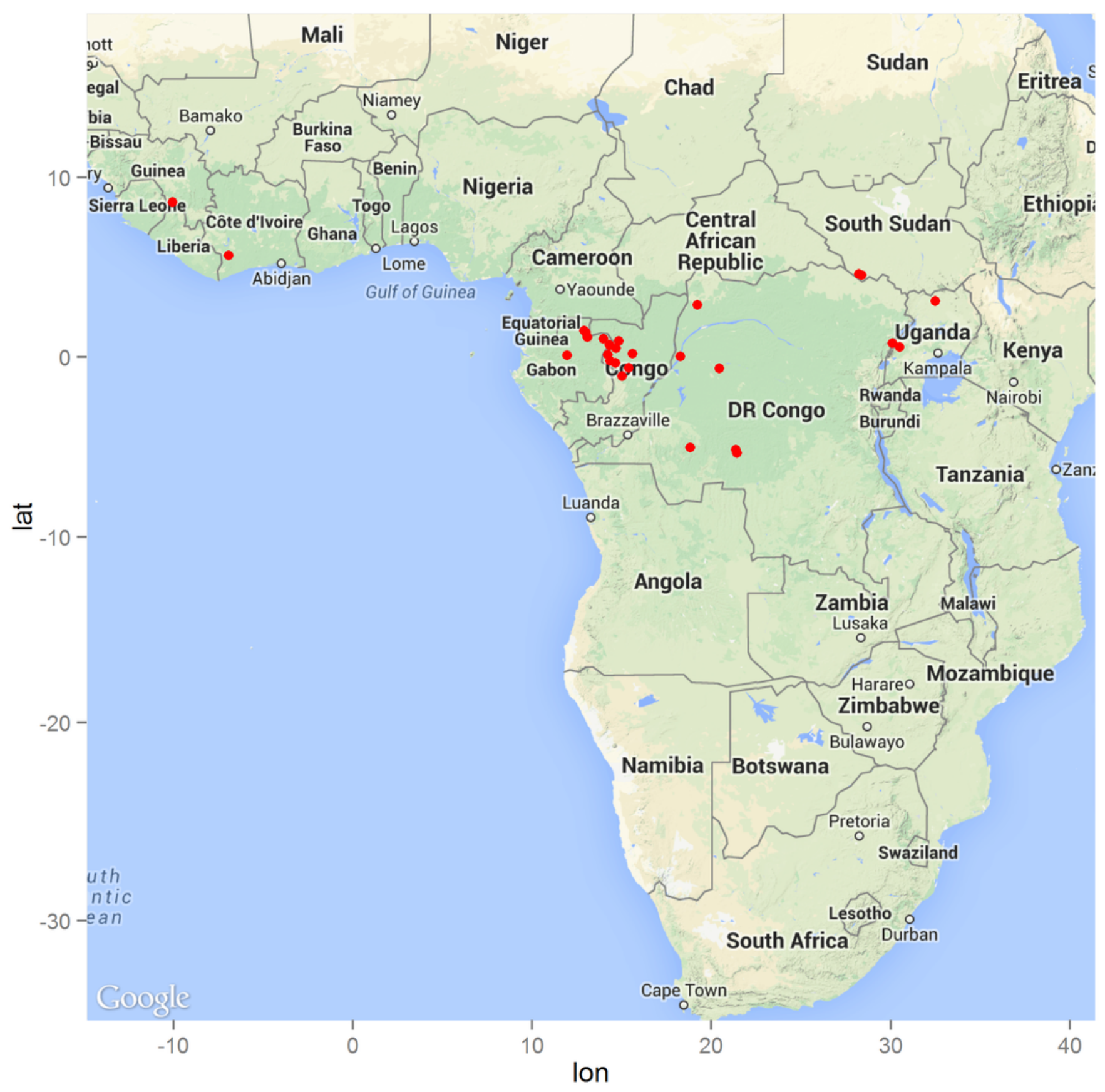




\section{2}

Figure 2. The distribution of mean annual temperature, altitude, maximum green vegetation fraction, and population density across the African continent with the distribution of zoonotic Ebola virus disease transmission events overlaid (red/blue dots).

In the map of population density, darker shades indicate higher population density, while areas of white indicate a population density less than 10 persons per $\mathrm{km}^{2}$. These maps depict the raster data distributions of the predictor variables and the observed point process of EVD spillover, rather than modeled associations. 

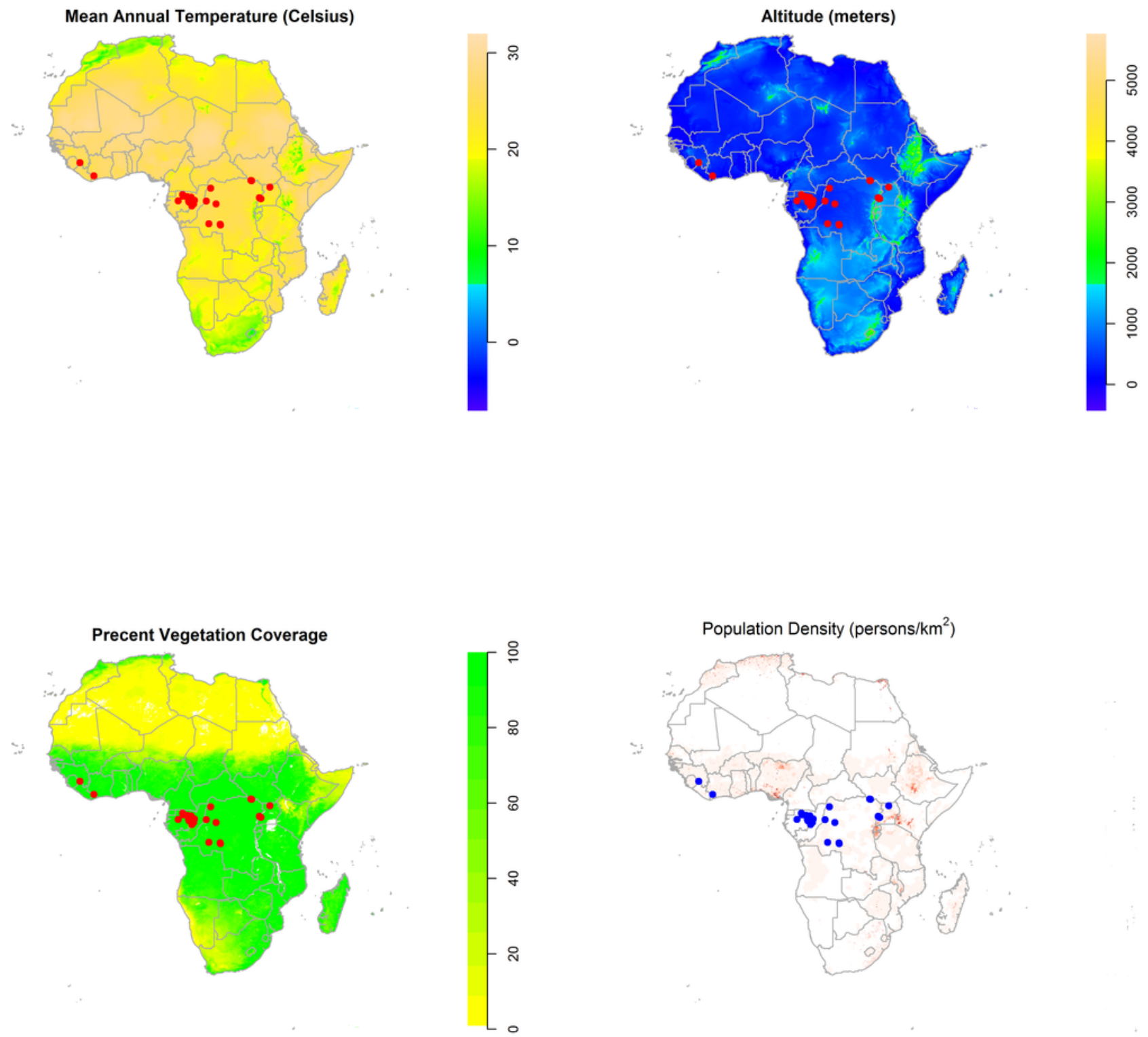
3

Predicted intensity of zoonotic Ebola virus disease transmission in West and Central Africa based on the modeled inhomogeneous Poisson point process.

Areas of predicted zoonotic transmission events are depicted in red, while the observed points of zoonotic transmission are depicted by blue dots.

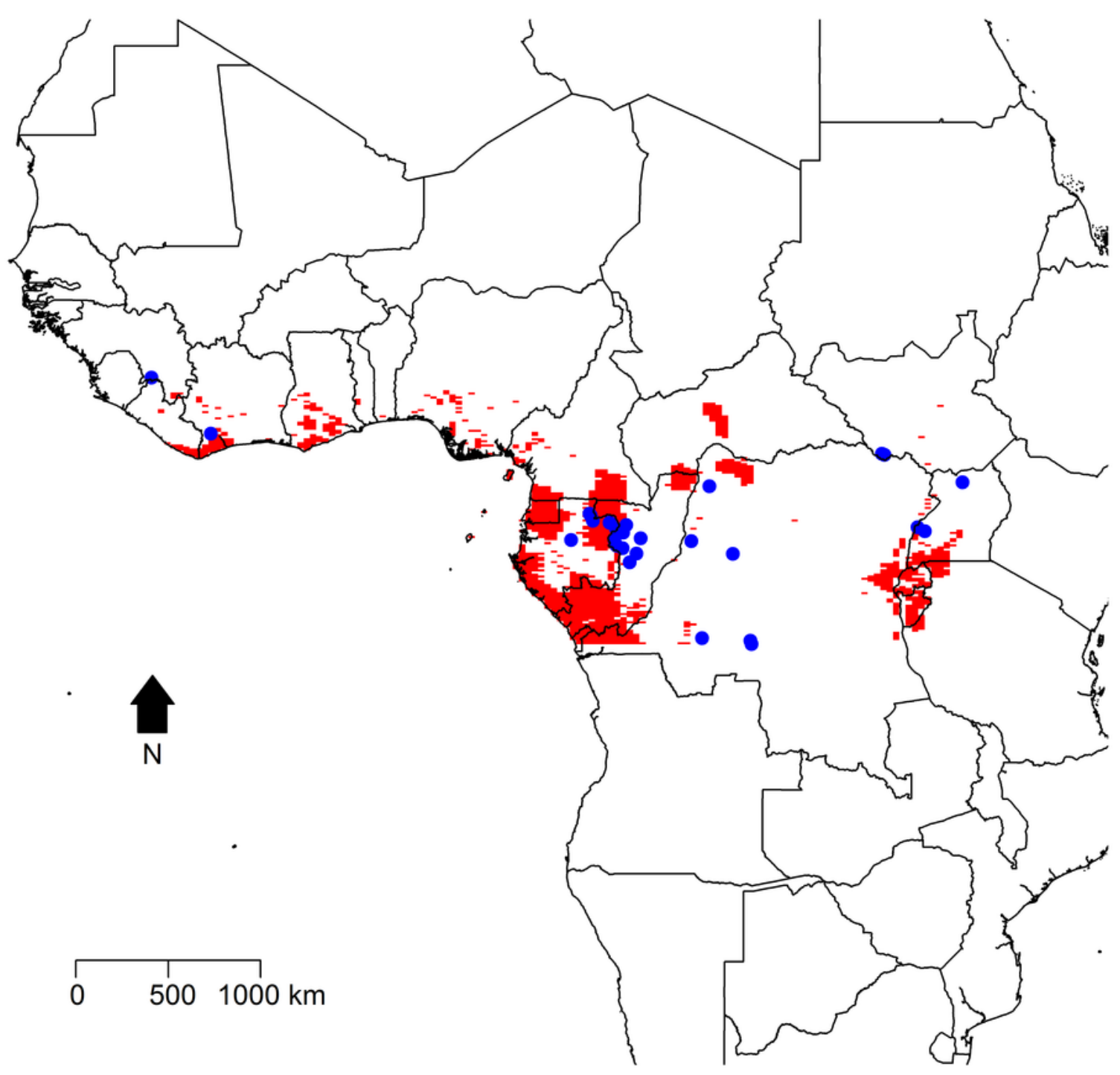

